\title{
One-step Generation of Zebrafish Carrying a Conditional Knockout-Knockin Visible Switch via CRISPR/Cas9-Mediated Intron Targeting
}

Jia $\mathrm{Li}^{1,5^{*}}$, Hong-yu $\mathrm{Li}^{1,2,5}$, Shan-ye $\mathrm{Gu}^{3,5}$, Hua-Xing $\mathrm{Zi}^{1,2}$, Lai Jiang ${ }^{3}$, and Jiu-lin $\mathrm{Du}^{1,2,4,6^{*}}$

${ }^{1}$ Institute of Neuroscience, State Key Laboratory of Neuroscience, CAS Center for Excellence in Brain Science and Intelligence Technology, Shanghai Research Center for Brain Science and Brain-Inspired Intelligence, Chinese Academy of Sciences, Shanghai 200031, China

${ }^{2}$ University of Chinese Academy of Sciences, Beijing 100049, China

${ }^{3}$ Department of Anesthesiology and Surgical Intensive Care Unit, Xinhua Hospital, Shanghai Jiao Tong University School of Medicine, Shanghai 200092, China

${ }^{4}$ School of Life Science and Technology, ShanghaiTech University, Shanghai 200031, China

${ }^{5}$ These authors contributed equally

${ }^{6}$ Lead Contact

*Correspondence: forestdu@ion.ac.cn, lijia@ion.ac.cn 


\section{SUMMARY}

The zebrafish has been becoming a popular vertebrate animal model in biomedical research. However, it is still challenging for making conditional gene knockout (CKO) zebrafish due to the low efficiency of homologous recombination (HR). Here we report an efficient non-HR-based method for generating zebrafish carrying a CKO and knockin (KI) switch (zCKOIS) coupled with dual-color fluorescent reporters. Using this strategy, we generated $h e y 2^{z \text { CKOIS }}$ which served as a hey2 KI reporter with EGFP expression. Upon Cre induction in targeted cells, the hey $2^{z \text { CKOIS }}$ was switched to a non-functional CKO allele hey $2^{z \text { CKOIS-inv }}$ associated with TagRFP expression, enabling to visualize CKO alleles. Thus, the simplification of the design, and the visibility and combination of both CKO and KI alleles' engineering make our zCKOIS strategy an applicable CKO approach for zebrafish.

\section{Keywords}

NHEJ, non-HR, knockin, conditional knockout, visible switch, zCKOIS, zebrafish

\section{INTRODUCTION}

Knockin (KI) animals carrying exogenous sequences integrated at specific genomic loci are invaluable tools for biomedical research. To understand the role of lethal genes in post-embryonic functions, it is usual to use $\mathrm{KI}$ animals carrying two loxP insertions at interested genomic loci to generate conditional gene knockout (CKO) animals (Yu and Bradley, 2001). The zebrafish (Danio rerio) is a vertebrate animal model excellent for in vivo imaging of biological events. However, to knockin two loxP sites in the zebrafish genome is still a big challenge due to the unavailability of gene targeting techniques for zebrafish embryonic stem cells and the low efficiency of homologous recombination (HR) in fast-developing fertilized zebrafish eggs (Robles 
et al., 2011). Although HR-mediated KI was reported to insert exogenous sequences into the genome, such as the green fluorescent protein $(G F P)$, loxP, and gene-trap cassette, the HR efficiency is low (Sugimoto, et al., 2017; Hoshijima, et al., 2016; Zu, et al., 2013). This low efficiency of the gene targeting via HR is a bottleneck for generating $\mathrm{KI}$ and $\mathrm{CKO}$ alleles in zebrafish. We previously developed a non-HR-mediated efficient KI strategy without destroying targeted genes, in which a Cas9 target is selected in an intron and the donor plasmid is integrated into the targeted intron (Li, et al., 2015). This strategy has been used to efficiently make KI zebrafish and mouse (Lovett-Barron, et al., 2019; Mu, et al., 2019; Suzuki, et al., 2019; Li, et al., 2015).

Here we develop a novel method for making zebrafish $\underline{\mathrm{CKO}}$ and KI $\underline{\text { switch }}$ (zCKOIS) in one step based on our previously reported non-HR-mediated intron targeting, in which a floxed and invertible gene-trap cassette with an RNA slice acceptor is inserted in the intron. Although Cre-mediated switch of the gene-trap cassette was reported to make CKO zebrafish, it failed to label the wild-type cells and the knockout cells, making it impossible to distinguish which cells are the mutant (Sugimoto, et al., 2017). Whereas the advantage of our design is that we can see the wild-type cells and the mutant cells directly by colors: without Cre expression, the zCKOIS cassette will not be inverted and a KI reporter with green fluorescence will be transcripted under the control of the target gene's endogenous promoter; in the present of Cre, the cassette will be inverse, and the target gene will be destroyed, associated with the expression of a $\mathrm{KO}$ reporter with red fluorescence. Using our strategy, we generated a hey $2^{z C K O I S}$ fish line and observed green fluorescence (i.e., KI reporter) in various cell types, including glial cells, endothelial cells (ECs) and haematopoietic stem cells (HSCs), similar to the expression pattern of the endogenous hey2 in zebrafish and mice (Rowlinson and Gering, 2010; Satow, et al., 2001; Zhong, et al., 2001). By injecting Cre mRNA, we then validated that the inverted $h e y 2^{z \text { CKOIS }}\left(\right.$ hey $\left.2^{\text {zCKOIS-inv }}\right)$ coupled with red fluorescence (i.e., KO reporter) is a non-functional $\mathrm{KO}$ allele, because homogenous hey2 ${ }^{\text {ZCKOIS-inv/zCKOIS-inv }}$ exhibited severe 
defects in tail circulation of the dorsal aorta (DA), a typical phenotype of the hey 2 point mutant gridlock (Zhong, et al., 2001; Zhong, et al., 2000). Finally, we achieved EC-specific KO of hey2 by crossing the hey $2^{z C K O I S}$ with the KI line Ki(flk1-P2A-Cre), in which Cre expression is driven by the EC-specific promoter $f l k l$. We found that $\mathrm{CKO}$ of hey 2 in ECs did not cause defects in tail circulation, suggesting that the Hey2 plays a non-EC-autonomous role in regulating circulation development.

\section{REUSLTS}

\section{Generation of a hey $2^{z C K O I S}$ Zebrafish Line}

We selected hey2 to test our strategy. The Hey2, a transcriptional repressor known as an effector of Notch signaling, is broadly expressed in various cell types, including ECs, HSCs, and glial cells (Rowlinson and Gering, 2010; Satow, et al., 2001; Zhong, et al., 2001). We first made a hey2-GSG-P2A-EGFP KI donor based on our previous study (Li, et al., 2015). The glycine-serine-glycine (GSG) sequence enables efficient cleavage of the P2A (Wang, et al., 2015). Then we modified the EGFP KI donor by inserting in the left arm with a gene trap cassette, which includes an invertible splice acceptor site followed by a red fluorescent protein (TagRFP) via a P2A sequence for multicistronic expression (Sugimoto, et al., 2017) (Figure 1A). We

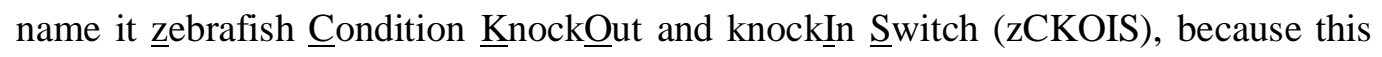
design is supposed to realize one-step generation of an allele with both EGFP-labeled KI and TagRFP-labeled KO. We co-injected the zCKOIS donor plasmid, a short guide RNA (sgRNA) and the mRNA of zebrafish codon-optimized Cas9 (zCas9) into one-cell-stage fertilized eggs and raised these embryos to adulthood (Figure 1A). Those F0 were then outcrossed to wild-type (WT) zebrafish, and their F1 progenies were screened for EGFP signal. Two F0 founders with germline transmission were identified in a total of $21 \mathrm{~F} 0$ fish. We then used F1 embryos from one of the founders for the following experiments. PCR of genomic DNA and sequencing analysis of the F1 progenies confirmed the inheritance of the genomic integration of the F0 founder 
(Figure 1B and Figure S1A). Reverse transcription (RT)-PCR and sequencing also confirmed that EGFP was expressed with the endogenous hey2 transcripts (Figure 1C and Figure S1B). Hereafter, we refer this line as hey $2^{z \text { CKOIS }}$.

Consistent with the reported expression patterns of hey 2 in zebrafish and mice (Rowlinson and Gering, 2010; Satow, et al., 2001; Zhong, et al., 2001), hey2 ${ }^{z C K O I S}$ showed intensive expression of EGFP in glial cells and vascular ECs (Figures 1D-1G). Interestingly, EGFP was found to express in arteries, such as the basal communicating artery (BCA) and postcardinal communicating segment (PCS) in the brain, and the dorsal aorta (DA) and arterial intersegmental vessels (aISVs) in the trunk. However, in veins, such as the choroidal vascular plexus (CVP) in the brain, and the postcardinal vein (PCV) and venous ISVs (vISVs) in the trunk, EGFP expression was hardly detected (Figures $\mathbf{1 F}$ and 1G). This is consistent with the role of hey2 in the fate decision of arterial ECs (Korten, et al., 2013; Zhong, et al., 2001). Taking together, it indicates that the EGFP-labeled KI of hey $2^{z \text { CKOIS }}$ recaptures the endogenous expression pattern of hey2 in zebrafish.

\section{Characterization of the hey $2^{z C K O I S}$ Allele}

To characterize the hey $2^{z C K O I S}$ allele, we injected Cre recombinase mRNA into the

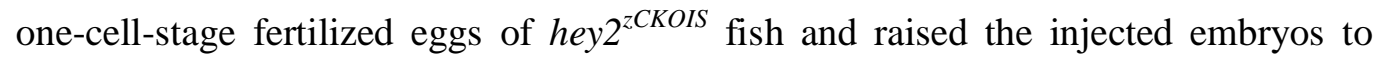
adulthood. By outcrossing to WT fish, we identified the F1 progenies with TagRFP

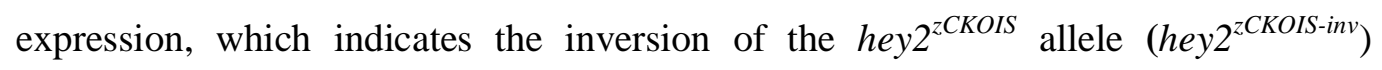
(Figures 2A and 2B). PCR and DNA sequencing of the F1 genomic DNA showed the inversion of the hey2 $2^{z C K O I S}$ cassette as expected (Figure 2C and Figure S2A). RT-PCR and sequencing data also confirmed the in-frame ligation of the mRNA of the hey2 exon4 to the P2A-TagRFP cassette (Figure 2D and Figure S2B).

We then imaged hey $2^{z \text { CKOIS-inv }} \mathrm{F} 1$ embryos and found that, similar to the EGFP expression pattern in hey $2^{z C K O I S}$ embryos, red fluorescence in hey $2^{z C K O I S-i n v}$ embryos was also expressed in glial cells and vascular ECs (Figures $\mathbf{2 E}$ and $\mathbf{2 F}$, and Figures S2C and S2D). Interestingly, we also observed that HSCs derived from ventral DA 
exhibited strong Hey2 expression during budding process (Figure 2G). In particular, we crossed hey $2^{z \text { CKOIS-inv }}$ with hey2 $2^{z \text { CKOIS }}$ and imaged the embryo of hey $2^{z \text { CKOIS/zCKOIS-inv }}$. The complete overlapping of green and red signals in the embryo indicates the $h e y 2^{z \text { CKOIS-inv }}$ faithfully recaptures the endogenous hey2 expression as the hey2 $2^{\text {CKOIS }}$ allele, implying that Cre-mediated inversion does not affect gene expression (Figures 2F and 2G, and Figures S2C and S2D). We noticed that in comparison with EGFP signal, TagRFP preferentially localized in the nuclear (Figures 2E-2G). The reason is probably that, the GSG spacer, which is crucial for the efficient cleavage of P2A, was added in the P2A-EGFP cassette but not the P2A-TagRFP cassette. This might cause the complete release of EGFP from Hey2, whereas the TagRFP might be partially fused with Hey2 and targeted to the nucleus where Hey2 is localized (Jia, et al., 2007).

The exon5 of hey 2 contains important functional domains of the Hey2 protein (Jia, et al., 2007). Cre-mediated inversion of TagRFP resulted in the skipping of the entire exon5 during mRNA processing (Figure 2B). Therefore, the hey $2^{z C K O I S-i n v}$ allele is supposed to be a loss-of-function of hey2. To validate it, we incrossed the $h e y 2^{z \text { CKOIS-inv/ }}$ fish and obtained homozygous hey $2^{z \text { CKOIS-inv/zCKOIS-inv }}$ embryos. Similar to the reported hey2 point mutant (i.e., gridlock) and KO zebrafish (Gibb, et al., 2018; Zhong, et al., 2000), these embryos exhibited pericardial edemas and loss of circulation to the tail (Figures $\mathbf{2 H}$ and 2I). These detects were not observed in the embryo of heterozygous hey $2^{z \text { CKOIS-inv/+ }}$, heterozygous hey $2^{z \text { CKOISv/+ }}$ or homozygous $h e y 2^{z \text { CKOIS/zCKOIS }}$ (Figure 2I), indicating that the hey2 $2^{\text {ZCKOIS }}$ is a functional KI allele and

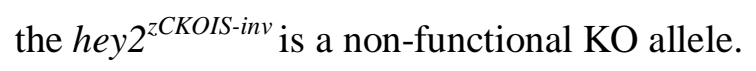

\section{EC-Specific KO of hey2}

Vascular ECs are important for supporting the normal function of the circulation system. Our data above showed that hey2 was strongly expressed in the DA and the $h e y 2^{z C K O I S-i n v / z C K O I S-i n v}$ exhibited gridlock phenotypes in the DA characterized by the loss of circulation to the tail (see Figures 1G and 2I). To test whether the loss of hey2 
in ECs is sufficient to cause the gridlock phenotype, we crossed the hey $2^{z \text { CKOIS }}$ fish to the KI line $\mathrm{Ki}(f l k 1-P 2 A-C r e)$, in which the Cre expression is under control of the EC-specific $f l k l$ promoter ( $\mathbf{L i}$, et al., 2015). By incrossing the fish of $h e y 2^{z C K O I S} ; K i(f l k 1-P 2 A-C r e)$, we obtained the progenies with the background of $h e y 2^{z C K O I S} ; K i(f l k 1-P 2 A-C r e)$ that both copies of the allele was supposed to be inverted and TagRFP should be specifically expressed in ECs (Figure 3A). Genotyping of the genomic DNA from these progenies confirmed the inversion of the hey $2^{z \text { CKOIS }}$ cassette (Figure S3A). RT-PCR data also confirmed the in-frame ligation of the mRNA of the hey2 exon4 to the P2A-TagRFP cassette (Figure 3B). As expected, TagRFP was observed in ECs of arteries, including the DA and aISVs, but not in ECs of the siblings without Cre induction (Figure 3C and Figure S3B), indicating the specific loss of the hey2 allele in ECs. As mentioned above, the hey2 $\mathrm{KO}$ displayed gridlock phenotype (see Figure 2I). Therefore, we examined whether EC-specific KO of hey2 could recapture the circulation defect and pericardial edemas. However, these phenotypes were not observed in the embryos with the genetic background of $h e y 2^{z \text { zKOIS/zCKOIS }} ; \mathrm{Ki}(f l k 1-P 2 A-C r e)$ (Figure 3D), suggesting that hey 2 may play a EC-independent role in regulating the circulation to the tail in zebrafish.

\section{DISCUSSION}

Here we provide a simple efficient method named zCKOIS for making zebrafish lines with both visible CKO and KI alleles in one step. We showed that the zCKOIS cassette can be targeted into the last intron of hey 2 with a high efficiency via CRIPSR/Cas9 mediated non-HR insertion. As the EGFP expression in hey $2^{\text {ZCKOIS }}$ embryos is driven by the endogenous hey2 promoter, EGFP was found to express in

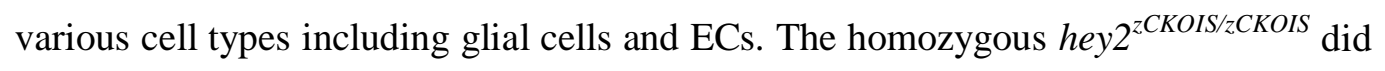
not display any gridlock phenotype, indicating that the hey $2^{z C K O I S}$ is a functional allele. Thus, the hey $2^{z C K O I S}$ can be used as a hey2 KI reporter with EGFP expression. On the other hand, by using Cre-induced inversion, we further validated that the 
TagRFP-tagged hey2 $2^{\text {ZKOIS-inv }}$ is a non-functional allele as the homozygous hey $2^{z \text { CKOIS-inv/ } z \text { CKOIS-inv }}$ larvae showed the obvious gridlock phenotype. Therefore, the $h e y 2^{z \text { CKOIS/zCKOIS }}$ fish with a tissue specific Cre expression can realize the CKO of the hey2 gene. Importantly, as the green color and red color represent the functional KI and non-functional $\mathrm{KO}$ alleles, respectively, we can easily distinguish $\mathrm{KO}$ cells and WT cells under microscopy just by eyes without sacrificing the fish for PCR and sequencing. This will greatly increase the screening efficiency and expand the way we detect $\mathrm{KO}$ events in vivo. For examples, we can make the inversion of the zCKOIS allele in some of the cells by using a line with weak Cre expression. Subsequently, cells with green (i.e., KI) or red color (i.e., KO) can be traced individually for exploring the distinct behaviors or the cell fate in a real-time manner in vivo.

For the successful one-step generation of zCKOIS of an interested gene, there are several key steps needed to be considered. The first one is how to choose a proper intron for the zCKOIS donor plasmid insertion. The ideal site for targeting is the intron just before the exon with important functional domains. The insertion of the $h e y 2^{z C K O I S}$ donor into the last intron of hey2 is because the last exon encodes important domains of the Hey2 protein, and thus the inversion of the DNA cassette in the hey $2^{z \text { CKOIS }}$ allele will result in the loss of the last exon during hey2 mRNA processing, guaranteeing the hey $2^{z C K O I S-i n v}$ is a non-functional $\mathrm{KO}$ allele. If the functional domain of an interested gene is present in the $X^{\text {th }}$ exon, the $(X-1)^{\text {th }}$ intron should be the suitable site for targeting. Second, it is important to select a sgRNA with a high cleavage efficiency in the intron. This is the crucial step for the successful insertion of the zCKOIS donor plasmid, because the highly efficient concurrent cleavage of the donor and target genomic sites will increase the germline transmission rate. For the case of the hey $2^{z C K O I S}$, the cleavage efficiency of the sgRNA used was about $50 \%$, resulting in successful identification of two founders among the total of 21 F0 fish. The third important step is the design of a zCKOIS donor. It is of importance to label the functional KI cassette and the KO cassette with different fluorescent colors, which will make it convenient to distinguish between the two 
genetic backgrounds by eyes. Last but not least, to achieve complete $\mathrm{CKO}$ in a specific cell type or tissue, the Cre expression needs to be highly specific. Leaky expression of Cre will potentially cause false-positive results. KI lines harboring the endogenous cis-regulatory elements of promoter/enhancer outperforms conventional transgenic lines in guaranteeing the specific expression ( $\mathbf{L i}$, et al., 2015). Therefore, we used the $\mathrm{Ki}(f l k l-P 2 A-C r e)$ line rather than the promoter-driven $T g(f l k l: C r e)$ transgenic line for the inversion of the zCKOIS allele in ECs. Unexpectedly, in the $h e y 2^{z \text { ZKOIS/zCKOIS }} ; \mathrm{Ki}(f l k 1-P 2 A-C r e)$, we did not observe the gridlock phenotype found in hey $\mathrm{KO}$ and gridlock mutants, suggesting that the hey 2 of ECs is not important for regulation of the circulation in the tail of zebrafish. A previous study showed that the gridlock phenotype can be suppressed by up-regulating vascular endothelial growth factor (VEGF) or activating the VEGF pathway (Peterson, et al., 2004). Therefore, it is possible that Hey2 promotes VEGF production in the surrounding cells. Then VEGF can be secreted into extracellular space and distributes around the DA to activate VEGF downstream signals in ECs for regulating the circulation to tail. Cell type-specific Cre mediated CKO with use of the hey2 $2^{z K O I S}$ line may help to find the detailed mechanisms in the future.

In summary, the method we introduced here realizes the generation of CKO and KI reporter line in one step via efficient non-HR-mediated insertion. The simplification and combination of CKO and KI make the zCKOIS strategy an applicable approach for zebrafish and even other organisms.

\section{EXPERMENTIAL PROCEDURES}

\section{Zebrafish Husbandry}

Adult zebrafish were maintained in the National Zebrafish Resources of China (Shanghai, China) with an automatic fish housing system at $28^{\circ} \mathrm{C}$. Embryos were raised under a $14 \mathrm{~h}-10 \mathrm{~h}$ light-dark cycle in $10 \%$ Hank's solution that consisted of (in $\mathrm{mM}$ ): $140 \mathrm{NaCl}, 5.4 \mathrm{KCl}, 0.25 \mathrm{Na}_{2} \mathrm{HPO}_{4}, 0.44 \mathrm{KH}_{2} \mathrm{PO}_{4}, 1.3 \mathrm{CaCl}_{2}, 1.0 \mathrm{MgSO}_{4}$ and 
4.2 $\mathrm{NaHCO}_{3}(\mathrm{pH}$ 7.2). Zebrafish handling procedures were approved by the Institute of Neuroscience, Chinese Academy of Sciences.

\section{Production of zCas9 mRNA, sgRNAs, and Cre mRNA}

The zCas9 expression plasmid pGH-T7-zCas9 (Liu, et al., 2014) was linearized by $X b a I$ and used as a template for Cas9 mRNA in vitro synthesis and purification with the mMACHINE T7 Ultra kit (Ambion). The coding sequence of Cre was amplified from Cre plasmid by using a T7-promoter included primers. The Cre mRNA was synthesized by the same T7 Ultra kit. The sequence of sgRNAs was designed according to previously reported criteria (Chang, et al., 2013). We used the CRISPR/Cas9 design tool (http://crispor.tefor.net/crispor.py) to select specific targets to minimize off-target effects. The sequences of designed sgRNAs are as follows:

hey2: GGAAGGATAATGGTTGGGT (forward strand);

$f l k 1$ : TCTGGTTTGGAAGGACACAG (forward strand);

gfap: GTGCGCAACACATAGCACCA (reverse strand);

A pair of oligonucleotides containing the sgRNA targeting sequence were annealed and cloned downstream of the T7 promoter in the PT7-sgRNA vector. The sgRNA was synthesized by the MAXIscript T7 Kit (Ambion) and purified by using the mirVana ${ }^{\mathrm{TM}}$ miRNA Isolation Kit (Ambion).

\section{Generation of the hey $2^{\text {CKOIS }}$ Zebrafish Line}

The GSG-P2A-EGFP fragment was ligated into the PMD-19-T by T-A cloning to form the T-GSG-P2A-EGFP vector. The left and right arms for hey2 were amplified by the KOD-PLUS Neo DNA polymerase from WT zebrafish genomic DNA. Then, the arms were ligated to the 5' and 3' regions of the GSG-P2A-EGFP fragment in the T-GSG-P2A-EGFP vector, respectively. The inverted cassette with loxP sites, splice acceptor, TagRFP and BGHpA was amplified from the pZwitch+1 plasmid. The resulting 1.8-kb DNA fragment was ligated to the left arm sequence via the ClaI restriction enzyme site to get the hey $2^{\text {CKOIS }}$ donor plasmid. Finally, the zCas9 mRNA, 
hey2 sgRNA, and hey2 $2^{\text {CKOIS }}$ donor plasmid were co-injected into one-cell-stage fertilized zebrafish eggs. Each embryo was injected with $1 \mathrm{nl}$ of solution containing $800 \mathrm{ng} / \mu \mathrm{l}$ zCas9 mRNA, $80 \mathrm{ng} / \mu \mathrm{l}$ sgRNA, and $15 \mathrm{ng} / \mu \mathrm{l}$ donor plasmid. To screen

hey $2^{\text {CKOIS }}$ founders, adult fish were crossed with $\mathrm{AB}$ wild-type (WT) zebrafish, the genomic DNA was extracted at 3 days post-fertilization (dpf), and the germline transmission was detected by PCR and imaging.

The left arm and right arm sequences of the donor plasmid were amplified from genomic DNA isolated from adult WT AB zebrafish using the following primers:

1) Left arm amplification primers:

Forward: 5'- CGAGGTACCCACTCGTCGACAAAACTAGGG -3' and

Reverse: 5'- CGAGGATCCAAACGCTCCCACTTCAGTTC -3'.

2) Right arm amplification primers:

Forward: 5'- CGAACCGGTTAAATGTTGGATTTAAATGT -3' and

Reverse: 5'- CGACTGCAGTAGGGTTTTAGCAGGCACCG -3'.

\section{RNA Preparation and First-Strand cDNA Synthesis}

Total RNAs of zebrafish embryos of $1.5 \mathrm{dpf}$ and $3.5 \mathrm{dpf}$ were extracted by using TRIzol reagent according to the manufacturer's instructions (Invitrogen, 15596018). The extracted total RNA was used to generate the first-strand cDNA by using PrimeScript ${ }^{\mathrm{TM}}$ RT Master Mix (Takara, RR036A).

Genotyping of $h e y 2^{z C K O I S}$ and $h e y 2^{z C K O I S-i n v}$ Genomic Loci and RT-PCR Analysis of the Transcripts

The following primers were used for PCR of genome DNA and RT-PCR of cDNA in Figures 1B and 1C, Figures 2C and 2D, Figure 3B, and Figure S3A.

F1: 5'- GATCTGCCAAGTTGGAGAAAGC -3'

F2: 5'-TCAATTAAGTTTGTGCCCCAGT -3'

R1: 5'-CACCGTGAACAACCACCACT -3'

R2: 5'-CTTGTACAGCTCGTCCATGCC -3' 
hey2 cDNA control RT-PCR primers:

F: 5'-ATGAAGCGGCCCTGTGAGGA - $3^{\prime}$ and

R: 5'-CTTTTCCTCCTGTGGCCTGAA -3'

\section{Generation of the KI Line Ki(flk1-P2A-Cre) with Cre Expression Specific in Vascular Endothelial Cells}

The Ki(flk1-P2A-Cre) line was generated with CRISPR/Cas9-mediated KI method as previously reported (Li, et al., 2015). Briefly, the flkl-P2A-Cre plasmid donor was made by replacing the EGFP sequence in the flk1-P2A-EGFP donor with Cre. $1 \mathrm{nl}$ of solution containing 800 pg zCas9 mRNA, 80 pg flkl gRNA and 15 pg flkl donor plasmid, was injected into zebrafish embryos at one-cell stage. The embryos were raised to adulthood for founder screening. To screen $\mathrm{Ki}(f l k 1-\mathrm{P} 2 \mathrm{~A}-\mathrm{Cre})$ founders, adult fish were crossed with $\mathrm{AB}$ WT zebrafish, the genomic DNA was extracted at $1 \mathrm{dpf}$, and the germline transmission was detected by PCR. The founder was then crossed to the lineage tracing line $T g$ (bactin2:loxP-STOP-loxP-DsRed-express) ${ }^{\text {sdl }}$ to confirm the Cre-mediated loxP site deletion in vessels (Figure S3C) (Bertrand, et al., 2010).

\section{Generation of the Glial Reporter KI Line Ki(GFAP-TagBFP)}

The $K i(G F A P-T a g B F P)$ line was generated as previously reported (Li, et al., 2015). Briefly, The GFAP-TagBFP donor was made by replacing the EGFP sequence in the GFAP-EGFP donor with TagBFP. $1 \mathrm{nl}$ of solution containing 800 pg zCas9 mRNA, 80 pg gfap gRNA and $15 \mathrm{pg}$ donor plasmid, was injected into zebrafish embryos at one-cell stage. The embryos were raised to adulthood for founder screening. To screen $K i(G F A P-T a g B F P)$ founders, adult fish were crossed with WT zebrafish, the genomic DNA was extracted at $1 \mathrm{dpf}$, and the germline transmission was detected by PCR and imaging.

\section{Confocal Imaging}

Z-stack images were taken at room temperature, under an Apo LWD 25X 
water-immersion objective (N.A., 1.1) by using a Nikon FN1 confocal microscope (Nikon, Japan). The z-step of images ranged from $3 \mu \mathrm{m}-5 \mu \mathrm{m}$. To detect pericardial edemas, a Plan Fluor 10x Water objective lens (N.A.0.3) was taken with a z-step of 5 $\mu \mathrm{m}$. The resolution of all the images was either $1024 \times 1024$ pixels or $512 \times 512$ pixels. Raw images were processed with Triangle method (ImageJ) for adjusting threshold.

\section{SUPPLEMENTAL INFORMATION}

Supplemental Information includes three figures.

\section{AUTHOR CONTRIBUTIONS}

J.L. and J.L.D. conceived the project and designed the experiments. J.L., H.Y.L. and S.Y.G. performed research and analyzed data. H.X.Z. made the Ki(flk1-P2A-Cre) zebrafish line. J.L., H.Y.L. and J.L.D. wrote the paper.

\section{ACKNOWLEDGEMENTS}

We are grateful to Drs. N Lawson for providing the $T g(f l k 1: E G F P)$ line, D Traver for providing the $\operatorname{Tg}$ (bactin2:loxP-STOP-loxP-DsRedEx) line and $\mathrm{K}$ Kikuchi for providing the $\mathrm{pZwitch}+1$ plasmid. This work was supported by the Young Scientists Fund of the National Natural Science Foundation of China (Grant No.31500849), Shanghai Municipal Science and Technology Major Project (18JC1410100, 2018SHZDZX05), the Key Research Program of Frontier Sciences (QYZDY-SSW-SMC028), the Strategic Priority Research Program (XDB32010200)

of Chinese Academy of Science, the International Partnership Program, Bureau of International Co-operation of Chinese Academy of Science(153D31KYSB20170059), China Wan-Ren Program, and Shanghai Leading Scientist Program. 


\section{DECLARATION OF INTERESTS}

The authors declare no competing interests.

\section{REFERENCES}

Bertrand, J.Y., Chi, N.C., Santoso, B., Teng, S., Stainier, D.Y., and Traver, D. (2010). Haematopoietic stem cells derive directly from aortic endothelium during development. Nature 464, 108-11.

Chang, N., Sun, C., Gao, L., Zhu, D., Xu, X., Zhu, X., Xiong, J.W., and Xi, J.J. (2013). Genome editing with RNA-guided Cas9 nuclease in zebrafish embryos. Cell research 23, 465-72.

Gibb, N., Lazic, S., Yuan, X., Deshwar, A.R., Leslie, M., Wilson, M.D., and Scott, I.C. (2018). Hey2 regulates the size of the cardiac progenitor pool during vertebrate heart development. Development 145.

Hoshijima, K., Jurynec, M.J., and Grunwald, D.J. (2016). Precise Editing of the Zebrafish Genome Made Simple and Efficient. Dev Cell 36, 654-67.

Jia, H., King, I.N., Chopra, S.S., Wan, H., Ni, T.T., Jiang, C., Guan, X., Wells, S., Srivastava, D., and Zhong, T.P. (2007). Vertebrate heart growth is regulated by functional antagonism between Gridlock and Gata5. Proc Natl Acad Sci U S A 104, 14008-13.

Korten, S., Brunssen, C., Poitz, D.M., Grossklaus, S., Brux, M., Schnittler, H.J., Strasser, R.H., Bornstein, S.R., Morawietz, H., and Goettsch, W. (2013). Impact of Hey 2 and COUP-TFII on genes involved in arteriovenous differentiation in primary human arterial and venous endothelial cells. Basic Res Cardiol 108, 362.

Li, J., Zhang, B.B., Ren, Y.G., Gu, S.Y., Xiang, Y.H., and Du, J.L. (2015). Intron targeting-mediated and endogenous gene integrity-maintaining knockin in zebrafish using the CRISPR/Cas9 system. Cell Res 25, 634-7.

Liu, D., Wang, Z., Xiao, A., Zhang, Y., Li, W., Zu, Y., Yao, S., Lin, S., and Zhang, B. (2014). Efficient gene targeting in zebrafish mediated by a zebrafish-codon-optimized 
cas9 and evaluation of off-targeting effect. Journal of genetics and genomics $=\mathrm{Yi}$ chuan xue bao 41, 43-6.

Lovett-Barron, M., Chen, R., Bradbury, S., Andalman, A.S., Wagle, M., Guo, S., and Deisseroth, K. (2019). Multiple overlapping hypothalamus-brainstem circuits drive rapid threat avoidance. bioRxiv.

Mu, Y., Bennett, D.V., Rubinov, M., Narayan, S., Yang, C.T., Tanimoto, M., Mensh, B.D., Looger, L.L., and Ahrens, M.B. (2019). Glia Accumulate Evidence that Actions Are Futile and Suppress Unsuccessful Behavior. Cell 178, 27-43 e19.

Peterson, R.T., Shaw, S.Y., Peterson, T.A., Milan, D.J., Zhong, T.P., Schreiber, S.L., MacRae, C.A., and Fishman, M.C. (2004). Chemical suppression of a genetic mutation in a zebrafish model of aortic coarctation. Nat Biotechnol 22, 595-9.

Rowlinson, J.M., and Gering, M. (2010). Hey2 acts upstream of Notch in hematopoietic stem cell specification in zebrafish embryos. Blood 116, 2046-56.

Satow, T., Bae, S.K., Inoue, T., Inoue, C., Miyoshi, G., Tomita, K., Bessho, Y., Hashimoto, N., and Kageyama, R. (2001). The basic helix-loop-helix gene hesr2 promotes gliogenesis in mouse retina. J Neurosci 21, 1265-73.

Sugimoto, K., Hui, S.P., Sheng, D.Z., and Kikuchi, K. (2017). Dissection of zebrafish shha function using site-specific targeting with a Cre-dependent genetic switch. Elife 6.

Suzuki, K., Yamamoto, M., Hernandez-Benitez, R., Li, Z., Wei, C., Soligalla, R.D., Aizawa, E., Hatanaka, F., Kurita, M., Reddy, P., et al. (2019). Precise in vivo genome editing via single homology arm donor mediated intron-targeting gene integration for genetic disease correction. Cell Res 29, 804-819.

Wang, Y., Wang, F., Wang, R., Zhao, P., and Xia, Q. (2015). 2A self-cleaving peptide-based multi-gene expression system in the silkworm Bombyx mori. Sci Rep $5,16273$.

Yu, Y., and Bradley, A. (2001). Engineering chromosomal rearrangements in mice. Nat Rev Genet 2, 780-90.

Zhong, T.P., Childs, S., Leu, J.P., and Fishman, M.C. (2001). Gridlock signalling 
pathway fashions the first embryonic artery. Nature 414, 216-20.

Zhong, T.P., Rosenberg, M., Mohideen, M.A., Weinstein, B., and Fishman, M.C. (2000). gridlock, an HLH gene required for assembly of the aorta in zebrafish. Science 287, 1820-4.

Zu, Y., Tong, X., Wang, Z., Liu, D., Pan, R., Li, Z., Hu, Y., Luo, Z., Huang, P., Wu, Q., et al. (2013). TALEN-mediated precise genome modification by homologous recombination in zebrafish. Nat Methods 10, 329-31. 


\section{FIGURE LEGENDS}

\section{Figure 1. Generation of a hey $2^{z C K O I S}$ Zebrafish Line.}

(A) Schematic of the intron targeting-mediated strategy for generating hey $2^{z \text { CKOIS }}$ zebrafish by using the CRISPR/Cas9 system. The zebrafish hey2 has 5 exons, and E4 and E5 represent the $4^{\text {th }}$ and $5^{\text {th }}$ exons, respectively. The hey2 $2^{z \text { CKOIS }}$ donor was integrated into the hey2 locus after co-injection of the donor with the sgRNA and zCas9 mRNA. The sgRNA target sequence is showed in red and the protospacer adjacent motif (PAM) sequence in green. The left and right arm sequences of the donor plasmids are indicated by the brown lines with double arrows. The left arm in the donor is $3300 \mathrm{bp}$ in length, including the original left arm in the genome and an inverted TagRFP cassette sequence. The right arm is $1107 \mathrm{bp}$ in length. GSG-P2A is glycine-serine-glycine-P2A sequence. (B) PCR analysis of the genomic DNA obtained from the $\mathrm{F} 1$ progenies of the hey $2^{z \text { CKOIS }}$ founder. A $3.2-\mathrm{kb}$ band was amplified by using the F1 and R1 primers, and a 5.3-kb band was amplified by using the F1 and R2 primers. The two bands are only present in hey $2^{z \text { CKOIS }}$ embryos, but not in the wild-type (WT) group. The F1, R1, F2, and R2 primers are indicated in (A). (C) Left, RT-PCR analysis using the cDNA of hey2 $2^{\text {CKOIS }} \mathrm{F} 1$ embryos. A $1.5-\mathrm{kb}$ band amplified by the $\mathrm{F} 1$ and R1 primers only appears in hey $2^{z C K O I S}$ embryos. Right, control RT-PCR using the primers binding hey2 coding sequence showed a $0.3-\mathrm{kb}$ band. (D) Projected confocal images (lateral view) of a hey $2^{\text {zKOIS }} ; \operatorname{Ki}($ GFAP-TagBFP) embryos at $1.5 \mathrm{dpf}$, showing EGFP expression in glial cells in the brain labeled by TagBFP. Ki(GFAP-TagBFP) is a KI line with TagBFP expression specific in glial cells. White arrowheads, the midbrain; Arrow, the forebrain. Scale bars: $100 \mu \mathrm{m}$. (E) Projected confocal images (lateral view) of a Ki(GFAP-TagBFP); hey $2^{z C K O I S}$ embryo at $3.5 \mathrm{dpf}$, showing EGFP expression in the glia cells at the spinal cord (white arrow heads). Cyan arrowheads, non-specific signals on the skin. Scale bars, $100 \mu \mathrm{m}$. (F and

G) Projected confocal imaging of the hey $2^{z C K O I S} ; T g(f l k 1$ :Ras-mCherry) embryos at 3.5 dpf. (F) EGFP expression in the basal communicating artery (BCA) and 
postcardinal communicating segment (PCS) in the brain (dorsal view). Top left, EGFP signal; top right, merged signals (EGFP/mCherry). The outlined areas labeled a and b in the top right panel are enlarged below. White arrowheads, BCA and PCS; White arrows, choroidal vascular plexus (CVP). (G) EGFP expression in the dorsal aorta (DA) but not in the postcardinal vein (PCV) in the trunk (lateral view). Arterial intersegmental vessels (aISVs) extending from the DA exhibited more EGFP signal than venous ISVs (vISVs) extending from the PCV. Top left, EGFP signal; top right, merged signals (EGFP/mCherry). The outlined areas labeled a and $\mathrm{b}$ in the top right panel are enlarged below. White arrowheads, DA and aISVs; White arrows, PCV and vISVs. Cyan arrowheads, non-specific signals on the skin. Scale bars: $100 \mu \mathrm{m}$.

\section{Figure 2. Characterization of the hey $2^{z \text { CKOIS-inv }}$ Allele.}

(A) Schematic of the hey $2^{z \text { CKOIS }}$ allele and the translated Hey $2^{\text {zCKOIS }}$ protein. The endogenous Hey2 protein includes an N-terminal bHLH domain, an Orange domain, and a protein-protein interaction YRPW ("Y") motif near the C-terminus. The Hey $2^{\text {zCKOIS }}$ protein is a fusion of the wild-type (WT) Hey2 protein and a GSG-P2A-EGFP. (B) Schematic of the hey $2^{z \text { ZKOIS-inv }}$ allele produced via Cre-induced inversion of the hey $2^{z \text { CKOIS }}$ allele. The translated Hey $2^{\text {zCKOIS-inv }}$ protein is a truncated Hey2 with only the bHLH domain fused to a P2A-TagRFP. (C) PCR detection of the inversion in hey $2^{z \text { CKOIS-inv }}$ genome. A 2.8 -kb band was only present in the hey $2^{z \text { CKOIS-inv }}$ but not in the WT or hey2 ${ }^{z C K O I S}$ embryos. (D) Left, RT-PCR analysis for detection of the transcription of hey2 $2^{\text {ZKOIS-inv }}$. A $0.9-\mathrm{kb}$ band was amplified only in the $h e y 2^{z \text { CKOIS-inv }}$, not in the WT or hey $2^{z C K O I S}$ embryos. Right, control RT-PCR using primers binding hey 2 coding sequence showed a $0.3-\mathrm{kb}$ band in all groups. (E) Projected confocal images (lateral view) of a hey $2^{z C K O I S-i n v} ; K i(G F A P-T a g B F P)$ embryo at $1.5 \mathrm{dpf}$, showing TagRFP expression in glia cells. The boxed area is enlarged on the right. White arrow heads, the midbrain; Arrow, the forebrain. Scale bars: $100 \mu \mathrm{m}$. (F)

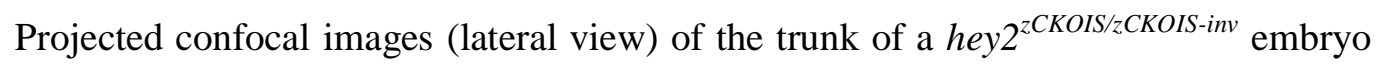
at $2.5 \mathrm{dpf}$. The red fluorescence is encoded by the truncated Hey2-P2A-TagRFP in the 
$h e y 2^{z C K O I S-i n v}$ allele and the green fluorescence is encoded by the WT Hey2-GSG-P2A-EGFP in the hey $2^{z C K O I S}$ allele. Cyan arrowheads, non-specific signals on the skin. Scale bars: $100 \mu \mathrm{m}$. (G) Confocal images of the DA in a

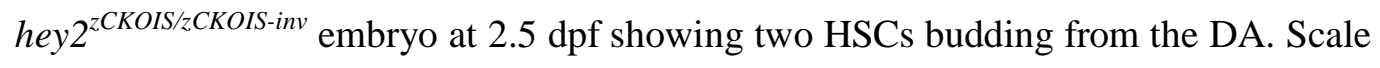
bars: $50 \mu \mathrm{m}$. (H) Bright-field images showing severe pericardial edema in the homozygous hey2 $2^{\text {ZCKOIS-inv/zCKOIS-in }}$ (top) but not in the heterozygous hey $2^{z \text { CKOIS-inv }}$ (bottom) at 3.5 dpf. Left, merged images of bright filed (BF) and TagRFP. Right, TagRFP. Scale bars: $500 \mu \mathrm{m}$. (I) Percentage of embryos with different genetic backgrounds showing normal circulation to the tail. The number on the bar is the total number of 3.5-dpf embryos examined.

\section{Figure 3. EC-Specific KO of hey2.}

(A) Schematic of the strategy for endothelial cell (EC)-specific KO of hey2. The Hey2 protein in ECs will be destroyed in the embryo carrying homozygous hey $2^{\text {ZCKOIS }}$ alleles and $\mathrm{Ki}(f l k 1-\mathrm{P} 2 \mathrm{~A}-\mathrm{Cre})$ background. In non-ECs where Cre is not expressed, the $h e y 2^{z C K O I S}$ will not be inverted and thus the WT Hey2 protein is expressed. (B) RT-PCR analysis using the cDNA for detection of the transcripts of hey $2^{z \text { CKOIS }}$ and hey $2^{z \text { CKOIS-inv }}$. Left, a $1.5-\mathrm{kb}$ band was amplified by the $\mathrm{F} 1$ and $\mathrm{R} 2$ primers in both $h e y 2^{z C K O I S} ; K i(f l k 1-P 2 A-C r e)$ and hey $2^{z C K O I S}$ but not in the WT embryos. Middle, a 0.9-kb band amplified by the F1 and F2 primers was only present in the $h e y 2^{z C K O I S} ; \mathrm{Ki}(f l k 1-\mathrm{P} 2 \mathrm{~A}-\mathrm{Cre})$ embryos. Right, control RT-PCR using primers binding hey 2 coding sequence showed a $0.3-\mathrm{kb}$ band in each group. (C) Projected confocal images (lateral view) of trunk vessels in a hey $2^{z C K O I S / 2 C K O I S} ; \mathrm{Ki}(f l k 1-\mathrm{P} 2 \mathrm{~A}-\mathrm{Cre}$ ) embryos at $3.5 \mathrm{dpf}$. Cre-induced inversion of the hey2 $2^{z C K O I S}$ enables the TagRFP expression in the DA and aISVs, where EGFP expression is barely deleted (arrowheads and dashed lines). Top left, EGFP signal; Top right, merged signals (EGFP/TagRFP). The outlined area is enlarged below. Cyan arrowheads, non-specific signals on the skin. Scale bars: $100 \mu \mathrm{m}$. (D) Percentage of the embryos with different genetic backgrounds showing normal circulation to the tail at $3.5 \mathrm{dpf}$. The number on the bar is the total number of 
bioRxiv preprint doi: https://doi.org/10.1101/827352; this version posted November 1 2019. The copyright holder for this preprint (which was not certified by peer review) is the author/funder, who has granted bioRxiv a license to display the preprint in perpetuity. It is made available under aCC-BY-NC-ND 4.0 International license.

fish examined. 
Endogenous hey2 locus

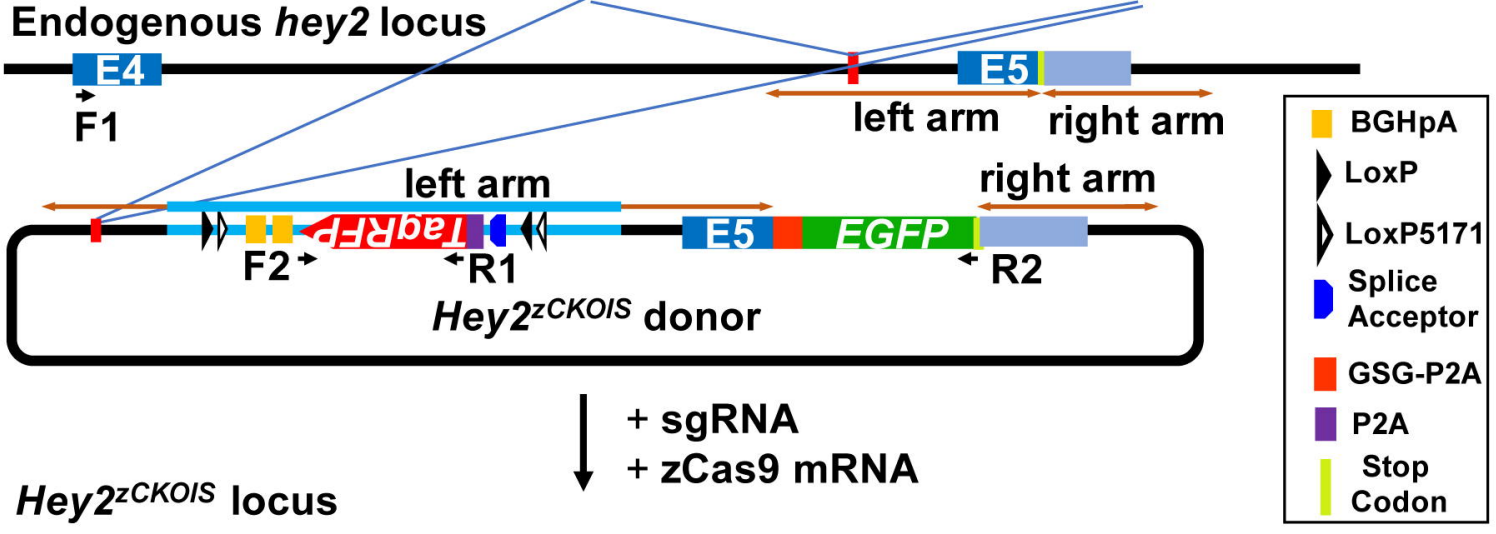

$\underset{\mathrm{F} 2}{\overrightarrow{\mathrm{R} 1}}$

B

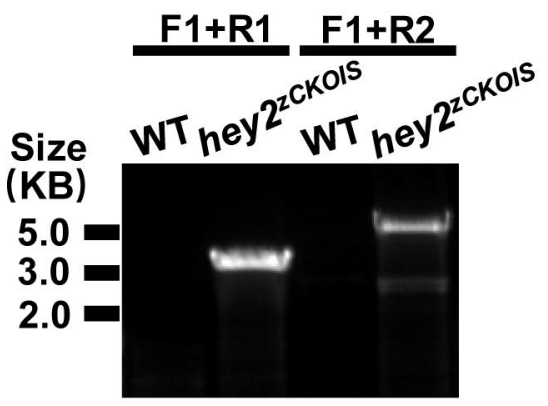

C

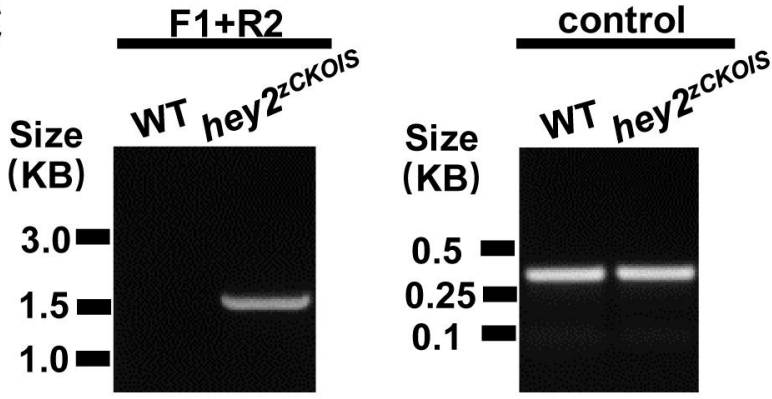

D

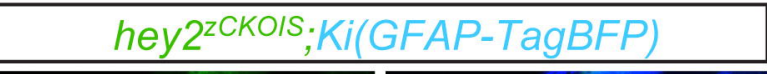

E

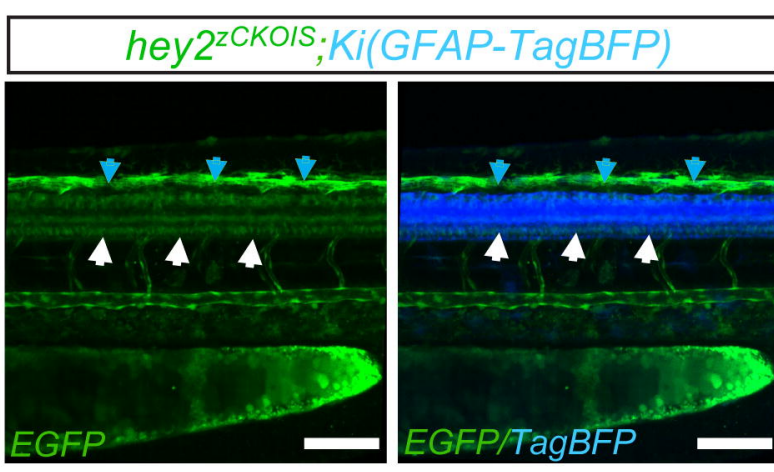

EGFP
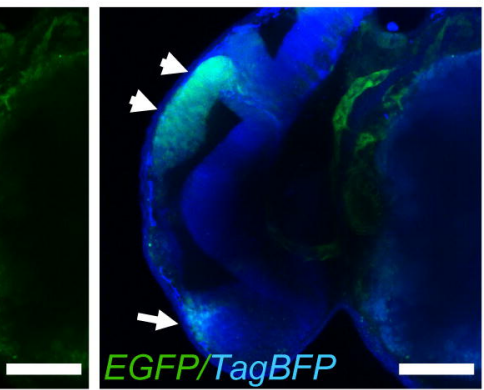

$\mathbf{F}$
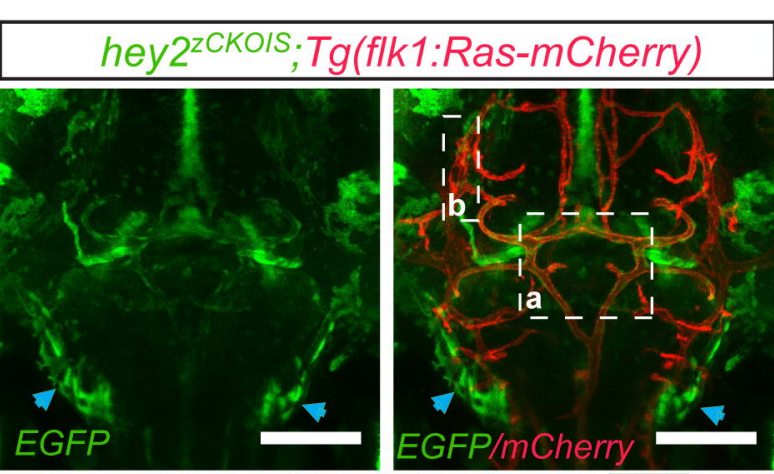

G
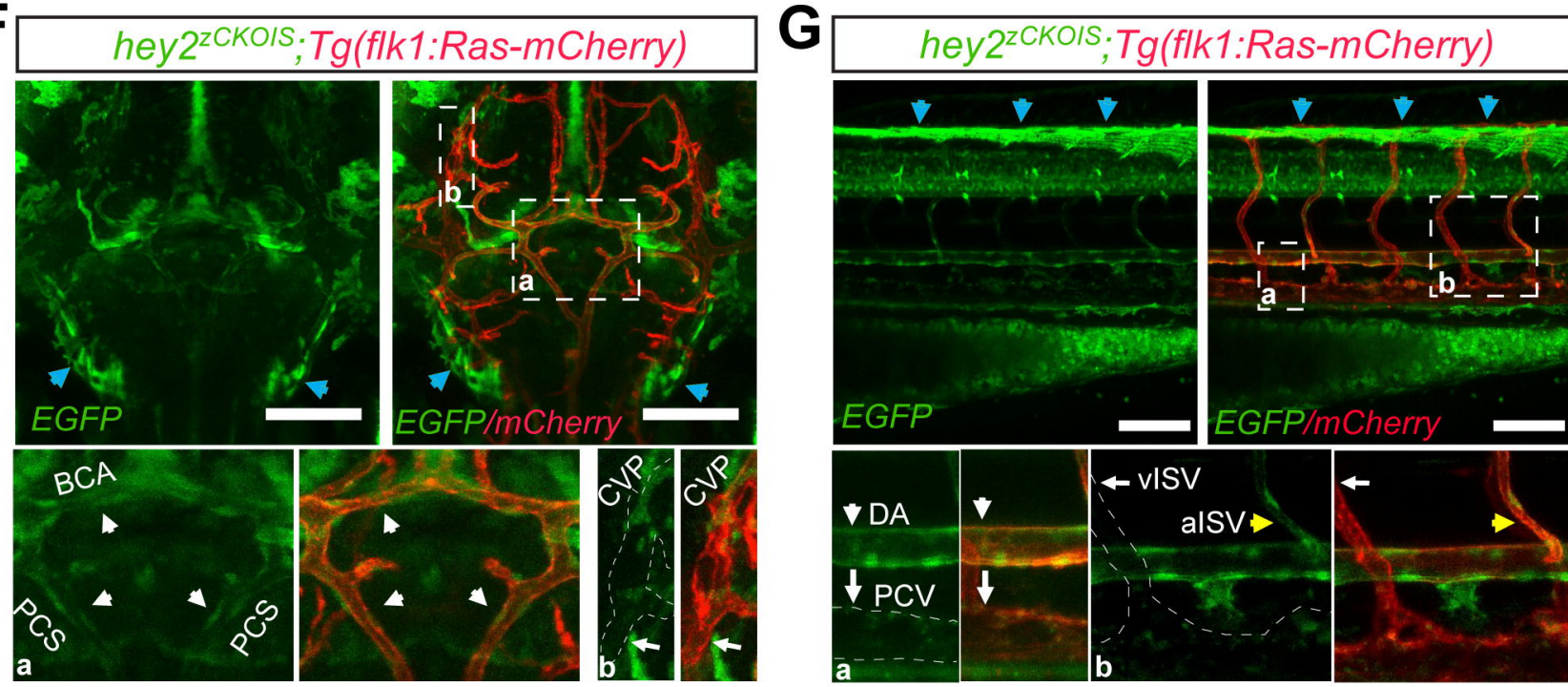

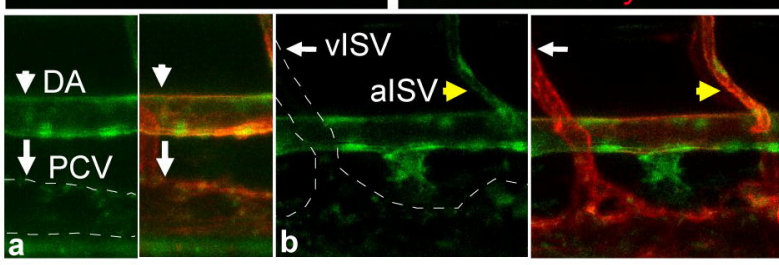


A hey2zckols

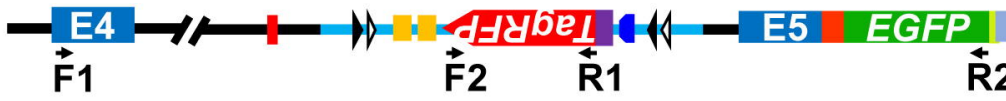

Hey2-GSG-P2A-EGFP bHLH Orange Y EGFP

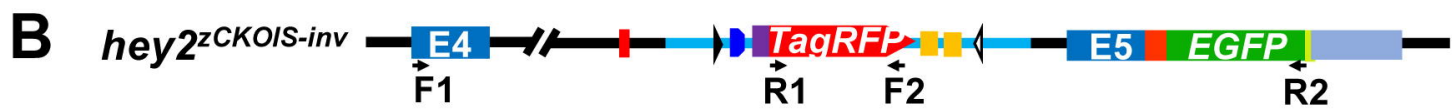
Hey2-P2A-TagRFP $\longrightarrow$ bHLH TagRFP

C

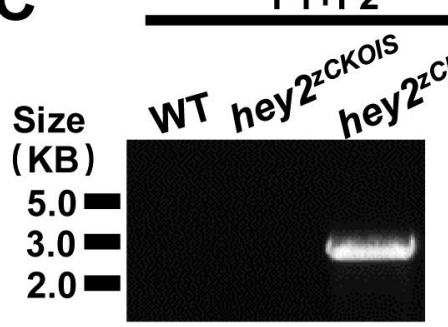

D

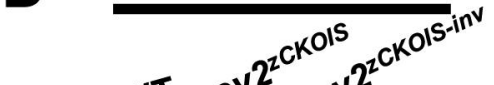

Size WT ney ${ }^{2{ }^{2}} \mathrm{y}^{2^{2}}$ Size (KB)

1.0

$0.5=$
(KB)

$0.5=$

0.25

$0.1=$ control

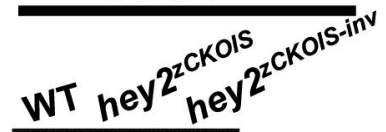

$\mathbf{E}$
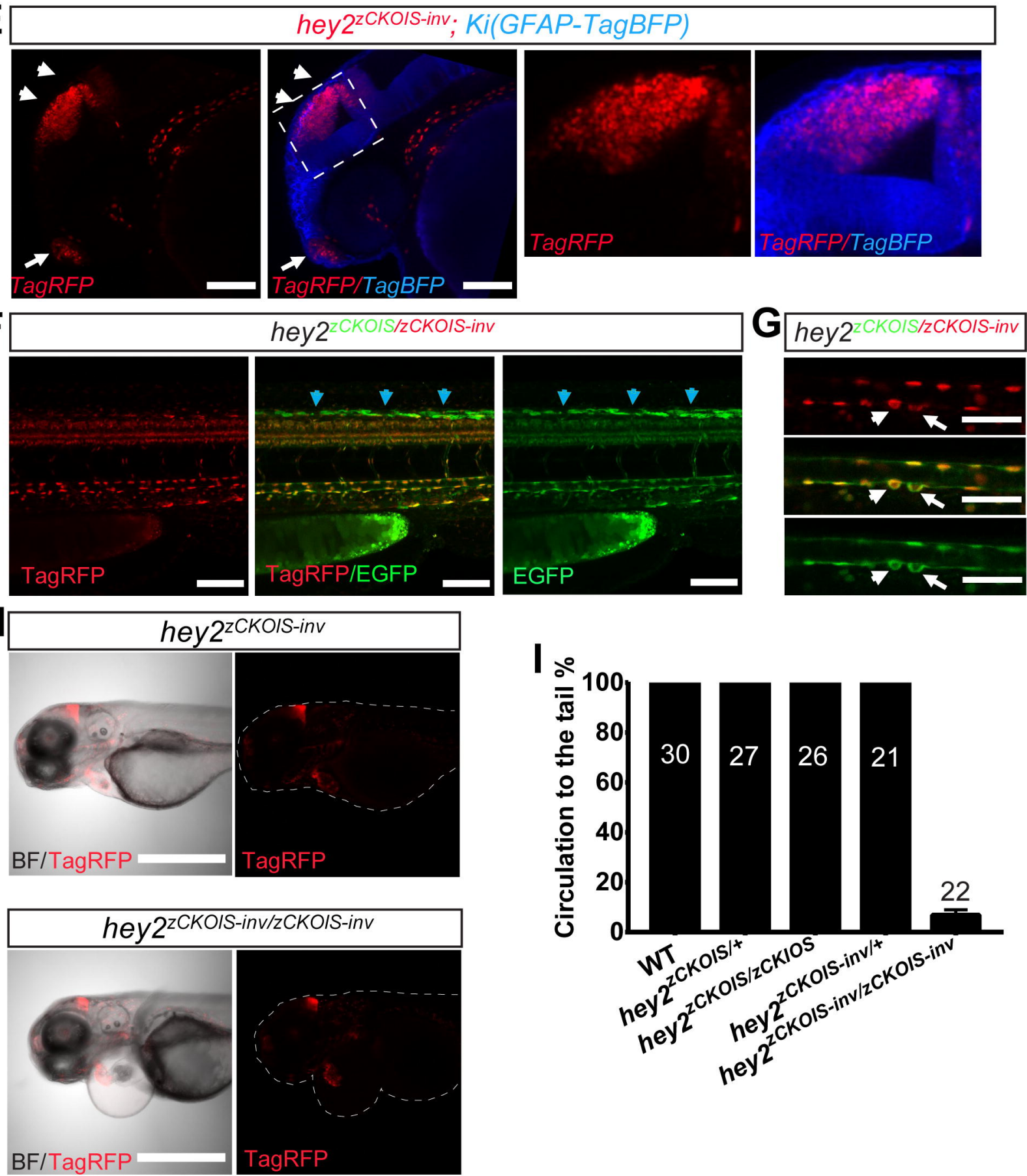

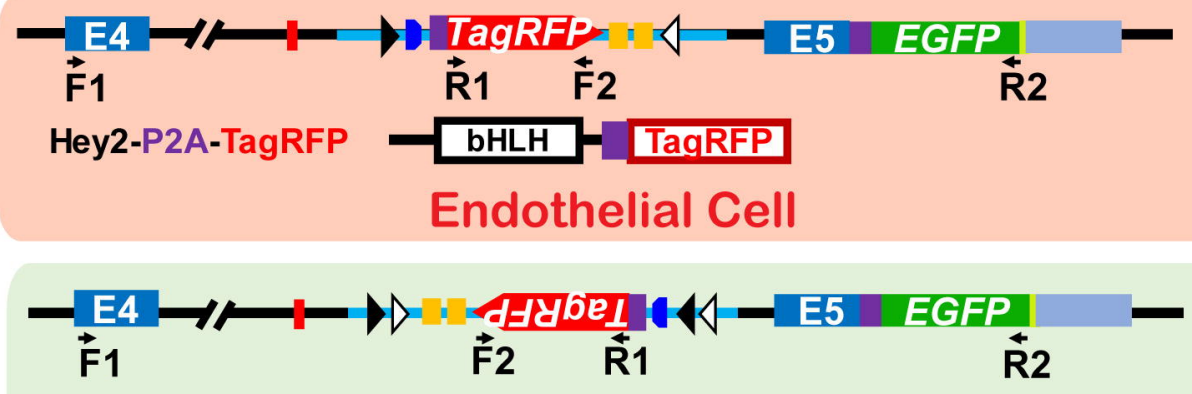
Hey2-GSG-P2A-EGFP $\mathrm{bHLH}$ Orange $\mathrm{Y}$ EGFP

Non-Endothelial Cell

B
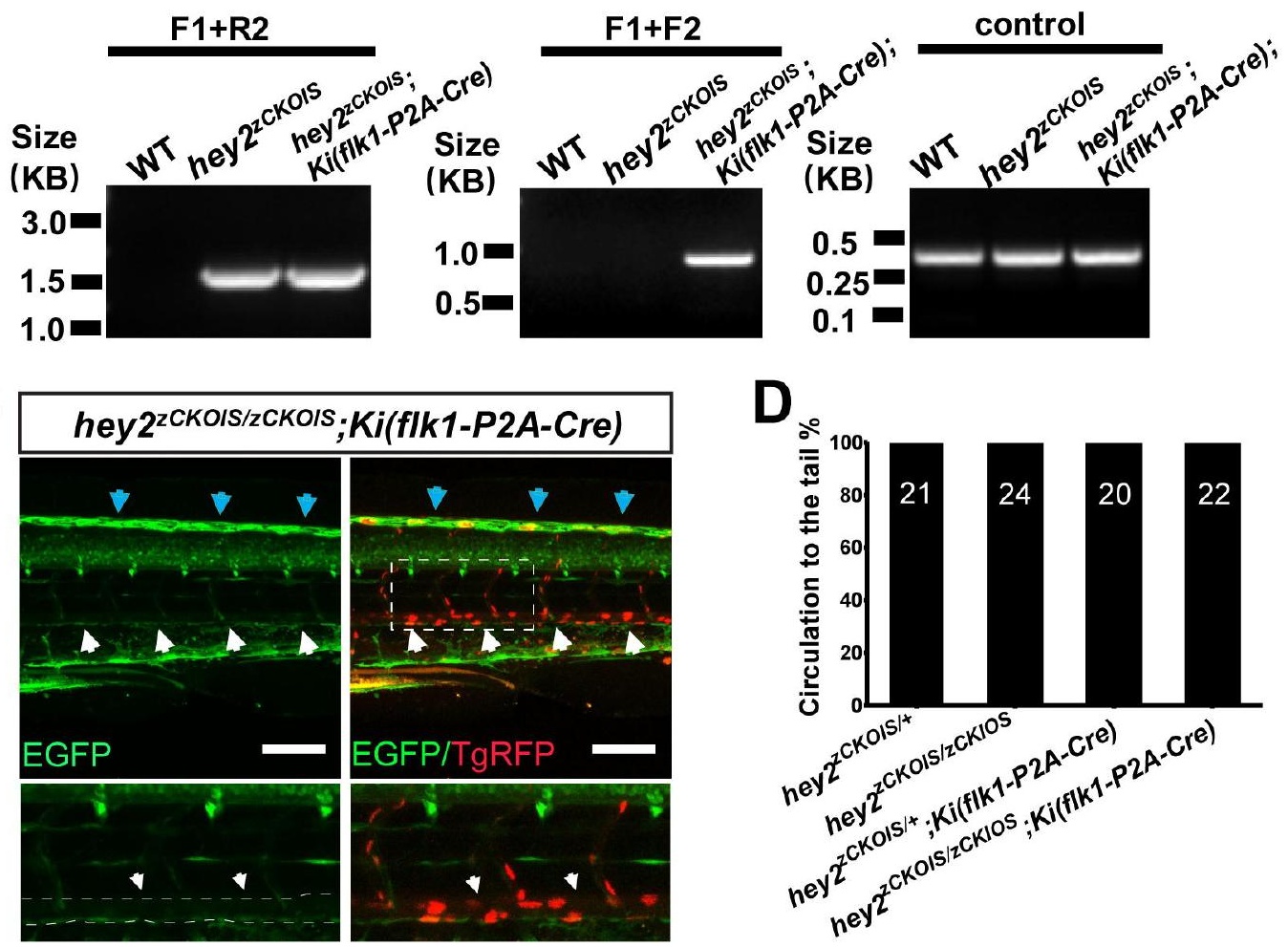

D

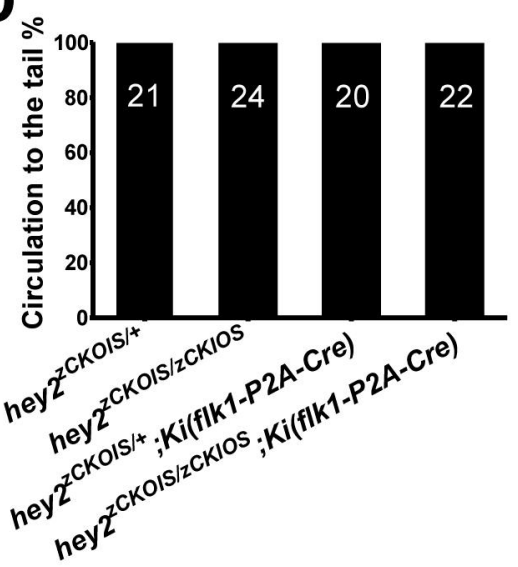

